\title{
BMJ open Protocol for a randomised clinical study comparing the effect of Roux-en-Y gastric bypass and sleeve gastrectomy on reactive hypoglycaemia in morbidly obese subjects
}

\author{
Geltrude Mingrone,${ }^{1}$ Simona Panunzi, ${ }^{2}$ Andrea De Gaetano, ${ }^{2}$ Caterina Guidone, ${ }^{1}$ \\ Marco Raffaelli, ${ }^{3}$ Cosimo Callari, ${ }^{3}$ Pio Celestino Lombardi, ${ }^{1}$ Rocco Bellantone ${ }^{3}$
}

To cite: Mingrone G, Panunzi S, De Gaetano A, et al. Protocol for a randomised clinical study comparing the effect of RouX-en-Y gastric bypass and sleeve gastrectomy on reactive hypoglycaemia in morbidly obese subjects. BMJ Open 2012;2:e002184. doi:10.1136/bmjopen-2012002184

- Prepublication history for this paper are available online. To view these files please visit the journal online (http://dx.doi.org/10.1136/ bmjopen-2012-002184).

Received 5 October 2012 Accepted 18 October 2012

This final article is available for use under the terms of the Creative Commons Attribution Non-Commercial 2.0 Licence; see

http://bmjopen.bmj.com

\section{${ }^{1}$ Department of Internal} Medicine, Catholic University, Rome, Italy

${ }^{2}$ National Research Council of Italy, Institute of Systems Analysis and Computer Science (IASI), BioMatLab, Rome, Italy

${ }^{3}$ Department of Surgery, Catholic University, Rome, Italy

Correspondence to: Professor Geltrude Mingrone; gmingrone@rm.unicatt.it

\section{ABSTRACT}

Introduction: Roux-en-Y gastric bypass (RYGB) is the most performed bariatric operation. Reactive hypoglycaemia is a frequent late complication occurring in about $72 \%$ of RYGB patients, which can present with various intensities up to the serious form of neuroglycopaenia. However, it seems to occur also after sleeve gastrectomy (SG) although much more rarely.

Methods and analysis: A single centre, open, 1-year randomised trial to compare the incidence of hypoglycaemia after RYGB or SG. A secondary objective is the assessment of the comparative ability of the two surgical procedures in determining the improvement or normalisation of insulin sensitivity, given the established relevance of insulin resistance in the cardiometabolic syndrome of obesity.

Ethics and dissemination: The study will be published and presented to international meetings and, due to the safety issue, it will represent a relevant information for national healthcare systems. The protocol was approved by the Catholic University Ethical Committee (A1534/CE/2012). Clinicaltrials.gov Registration n. NCT01581801.

\section{INTRODUCTION}

The overall prevalence of grade 2 and 3 adult obesity (body mass index, BMI $>35 \mathrm{~kg} /$ $\mathrm{m}^{2}$ ) derived from the 2009-2010 National Health and Nutrition Examination Survey (NHANES) exceeded $15 \%$ and grade 3 obesity (BMI $>40 \mathrm{~kg} / \mathrm{m}^{2}$ ) accounted for $6.3 \% .^{1}$ The dietary approach is of limited value in the short term and weight regain is practically the rule in the long run, as a consequence of the poor compliance to the diet shown by obese subjects. In fact, in a recent study, ${ }^{2}$ where different types of diets were assigned to a population of overweight and obese subjects (BMI from 25 to $40 \mathrm{~kg} / \mathrm{m}^{2}$ ), an

\section{ARTICLE SUMMARY}

Article focus

- To compare the incidence of reactive hypoglycaemia in sleeve gastrectomy with respect to Roux-en-Y gastric bypass, within 1 year after bariatric surgery.

- To assess the modifications in insulin sensitivity and secretion of these two bariatric operations.

Key messages

- Reactive hypoglycaemia is a late complication of gastric bypass, although it seems to occur also after sleeve gastrectomy. However, no study assessing the incidence of reactive hypoglycaemia after these operations is available in the literature. This randomised study aims at investigating the incidence of this complication and the changes in insulin sensitivity and secretion.

Strengths and limitations of this study

- The strength of this study is its randomised design. A limitation might be the fact that this is a short term study with 1 year time frame; however, in the secondary aim we foresee to follow the patients up to 5 years.

average of $6 \mathrm{~kg}$, corresponding to $7 \%$ of initial body weight, was lost in the first 6 months, but most of the weight was regained after 1 year and at 2 years, only $31-37 \%$ of the participants had maintained a weight loss of at least $5 \%$ of their initial weight.

Bariatric surgery has long been recognised as an effective treatment for grades 3 or 2 obesity associated with complications ${ }^{3}$ and, accordingly, the number of bariatric operations in the USA is growing over time from ca. 10000 in the early 1990s to 103000 in $2003 .{ }^{4}$

Bariatric surgery may determine type 2 diabetes remission, ${ }^{5}{ }^{6}$ while improving several other serious comorbidities. ${ }^{7}$ Among bariatric 
surgery procedures, Roux-en-Y gastric bypass (RYGB) was shown to account for $41 \%$ of all bariatric operations at least in the USA. ${ }^{8}$ Sleeve gastrectomy (SG), which was originally conceived as a first step before performing RYGB or biliopancreatic diversion with duodenal switch in super-obese patients, ${ }^{9}$ has recently gained a place as first-choice restrictive bariatric procedure. ${ }^{10}$ It was noted that SG determines weight loss similar to that achieved after $\mathrm{RYGB}^{11}$ and larger than that following laparoscopic adjustable gastric banding. ${ }^{12} 13$

Reactive hypoglycaemia is a late complication of RYGB, although it seems to occur also after SG.

After RYGB, early insulin secretion is enhanced during an oral glucose tolerance test $\left(\right.$ OGTT) ${ }^{14}$ or after a meal, ${ }^{15} 16$ which fact might explain the later reactive hypoglycaemia. An increasing number of reports highlight the occurrence of severe hypoglycaemia after RYGB, including cases of neuroglycopaenia attributed to nesidioblastosis. ${ }^{17-20}$ Roslin $e t a l^{21}$ found that $72 \%$, that is, 26 of 36 patients who underwent RYGB 6 months earlier, had reactive hypoglycaemia at $2 \mathrm{~h}$ during OGTT ( $100 \mathrm{~g}$ of glucose), which was defined as 'an absolute serum glucose level $\leq 60 \mathrm{mg} / \mathrm{dl}$, or a drop of $100 \mathrm{mg} / \mathrm{dl}$ in serum glucose level in $1 \mathrm{~h}$ '.

As compared with RYGB, SG seems to have a much lower occurrence of reactive hypoglycaemia, ca. 3\%, that is, 1 of 31 patients studied at 6 weeks after the operation in one series. ${ }^{22}$

Fortunately, the frequency of severe hypoglycaemia or related symptoms requiring hospitalisation after RYGB is pretty low, the adjusted HRs were in fact 2.7 for hypoglycaemia, 2.8 for confusion, 4.9 for syncope, 3.0 for epilepsy and 7.3 for seizures in a Swedish retrospective cohort study on 5040 cases based on national registries. ${ }^{23}$

However, until now no prospective studies have investigated the incidence of hypoglycaemia after RYGB and no randomised studies have been undertaken to compare the effect of SG to that of RYGB in terms of incidence of hypoglycaemic episodes.

The primary aim of the present study was to conduct a 1-year randomised trial to compare the incidence of hypoglycaemia after RYGB or SG. A secondary objective of great interest is the assessment of the comparative ability of the two surgical procedures in determining the improvement or normalisation of insulin sensitivity in this class of subjects, given the established relevance of insulin resistance in the cardiometabolic syndrome.

\section{STUDY OBJECTIVE}

\section{Primary objective}

The primary objective of the study is to compare the incidence of reactive hypoglycaemia in SG with respect to RYGB, within 1 year after bariatric surgery.

\section{Secondary objectives}

- To quantify the relative contribution of changes in insulin sensitivity and insulin secretion during an OGTT on the glycaemic effects of bariatric surgery, and to determine if differences exist between the two surgical treatments.

- To determine whether treatment is associated with body weight, BMI and abdominal circumference loss, with body composition changes, as assessed by DEXA (dual-energy x-ray absorptiometry), and with lipid profile modifications.

- To compare between SG and RYBG the incidence of severe hypoglycaemia or related symptoms (shakiness, sweating, dizziness or light-headedness, difficulty speaking, weakness, confusion, syncope, epilepsy and seizures) within 5 years after surgery.

\section{EXPERIMENTAL DESIGN}

This is a randomised study of gastric bypass as compared with SG. Since there is no agreement and no consensus about the criteria for the selection of a certain type of bariatric operation in relation to other types, the randomisation does not have ethical implications.

The protocol was approved by the Catholic University Ethical Committee (A1534/CE/2012).

\section{Study endpoints}

\section{Primary endpoint}

The primary endpoint of the study is the incidence of reactive hypoglycaemia at 1 year after bariatric surgery.

\section{Secondary endpoints}

- Changes at 1 year in insulin sensitivity and insulin secretion during an OGTT.

- Changes at 1 year in body weight, BMI, abdominal circumference, body composition and lipid profile.

- Incidence of severe hypoglycaemia or related symptoms (shakiness, sweating, dizziness or lightheadedness, difficulty speaking, weakness, confusion, syncope, epilepsy and seizures) within 5 years after surgery.

\section{Experimental plan}

This is a monocentric prospective controlled randomised clinical trial designed to investigate the difference in incidence of reactive hypoglycaemia between SG and RYGB within 1 year after surgery.

At the moment of the screening visit subjects will be randomised to undergone either SG or RYGB.

\section{Study design diagram}

The diagram of the study plan is as follows: 


\begin{tabular}{|c|c|c|c|c|c|c|c|}
\hline & Visit 1 & Visit 2 & Visit 3 & Visit 4 & Visit 5 & Visit 6 & Visit 7 \\
\hline & $\begin{array}{l}\text { Screening } \\
\text { visit } \\
\text { (-3 months) }\end{array}$ & $\begin{array}{l}\text { Baseline } \\
\text { visit } \\
\text { (-1 month) }\end{array}$ & $\begin{array}{l}\text { Follow-up } \\
\text { visit } \\
\text { (+1 month) }\end{array}$ & $\begin{array}{l}\text { Follow-up } \\
\text { visit } \\
\text { (+3 months) }\end{array}$ & $\begin{array}{l}\text { Follow-up } \\
\text { visit } \\
\text { (+6 months) }\end{array}$ & $\begin{array}{l}\text { Follow-up } \\
\text { visit } \\
\text { (+9 months) }\end{array}$ & $\begin{array}{l}\text { Follow-up visit } \\
\text { (+12 months) }\end{array}$ \\
\hline $\begin{array}{l}\text { Written informed } \\
\text { consent }\end{array}$ & $\mathrm{x}$ & $\mathrm{x}$ & & & & & \\
\hline $\begin{array}{l}\text { Demographic } \\
\text { data }\end{array}$ & $x$ & & & & & & \\
\hline $\begin{array}{l}\text { Medical and } \\
\text { surgical history }\end{array}$ & $x$ & $x$ & & & & & \\
\hline $\begin{array}{l}\text { Physical } \\
\text { examination* }\end{array}$ & $x$ & $x$ & $x$ & $x$ & $x$ & $x$ & $x$ \\
\hline Height & $x$ & & & & & & \\
\hline Weight, BMI & $x$ & $x$ & $x$ & $x$ & $x$ & $x$ & $x$ \\
\hline $\begin{array}{l}\text { Waist and hip } \\
\text { circumference }\end{array}$ & $x$ & $x$ & $\mathrm{x}$ & $x$ & $\mathrm{x}$ & $\mathrm{x}$ & $x$ \\
\hline DEXA & & $x$ & & $x$ & $x$ & $x$ & $x$ \\
\hline $\begin{array}{l}\text { Blood pressure } \\
\text { and HR }\end{array}$ & $x$ & $x$ & $x$ & $x$ & $x$ & $x$ & $x$ \\
\hline ECG & $x$ & $x$ & $x$ & $x$ & $x$ & $\mathrm{x}$ & $x$ \\
\hline $\begin{array}{l}\text { Laboratory } \\
\text { assessments } †\end{array}$ & $x$ & $x$ & $\mathrm{x}$ & $x$ & $\mathrm{x}$ & $x$ & $x$ \\
\hline OGTT & $x$ & $x$ & $x$ & $x$ & $x$ & $x$ & $x$ \\
\hline $\begin{array}{l}\text { Adverse events } \\
(A E) \text { recording }\end{array}$ & & & $\mathrm{x}$ & $x$ & $x$ & $x$ & $x$ \\
\hline $\begin{array}{l}\text { Concomitant } \\
\text { medication }\end{array}$ & $\mathrm{x}$ & $x$ & $x$ & $x$ & $\mathrm{x}$ & $\mathrm{x}$ & $x$ \\
\hline
\end{tabular}

*See box 1 related to the physical examination and DEXA.

†See table 1 related to the Laboratory assessments.

An overview of all planned blood samples, including volumes and purpose is provided in table 1 .

\section{Study duration}

Subjects will be studied from the time of screening visit to 1 year after the surgical procedure. The study will undergo four distinct phases:

- Screening visit (month 3)

- Baseline visit (month 1)

- A follow-up period after the surgical treatment (1 year of duration, months 1, 3, 6, 9 and 12)

- A further limited yearly follow-up period after study conclusion (4 years of duration)

\section{Screening visit}

At month 3, eligible patients will be identified and the written informed consent for enrolment will be obtained. Demographic data, anthropometric measures, physical examination variables, information about medical history and concomitant medications, as well as variables related to inclusion criteria will be collected.

\section{Basal visit}

At month 1 enrolled patients will be asked to give their informed consent to randomisation. Study compliance will be assessed during the screening and baseline periods using attendance at appointments and completion of questionnaires. Baseline anthropometric measures, body composition, blood pressure and biochemical data (levels of fasting plasma glucose, glycated haemoglobin (HbA1c), C-peptide and serum insulin, lipid profile and OGTT) are measured.

\section{Follow-up period}

At 1 month after bariatric surgery and every 3 months until 1 year after surgery (hence at month 1, 3, 6,

Box 1 Variables recorded during the physical examination and included in DEXA

Physical examination

Appearance in general

Skin

Head and neck

Lymph nodes

Thyroid

Cardiovascular system

Respiratory systems

Abdomen

Concomitant medication

Other

DEXA

Total-body bone mineral density

Lean body mass

Fat mass

Percentage of weight body fat 


\begin{tabular}{|c|c|c|c|c|c|c|c|}
\hline & $\begin{array}{l}\text { Visit } 1 \\
\text { Screening } \\
\text { visit }\end{array}$ & $\begin{array}{l}\text { Visit } 2 \\
\text { Baseline } \\
\text { visit }\end{array}$ & $\begin{array}{l}\text { Visit } 3 \\
\text { Follow-up } \\
\text { visit } \\
\text { (+1 month) }\end{array}$ & $\begin{array}{l}\text { Visit } 4 \\
\text { Follow-up } \\
\text { visit } \\
\text { (+3 months) }\end{array}$ & $\begin{array}{l}\text { Visit } 5 \\
\text { Follow-up } \\
\text { visit } \\
\text { (+6 months) }\end{array}$ & $\begin{array}{l}\text { Visit } 6 \\
\text { Follow-up } \\
\text { visit } \\
\text { (+9 months) }\end{array}$ & $\begin{array}{l}\text { Visit } 7 \\
\text { Follow-up } \\
\text { visit } \\
\text { (+12 months) }\end{array}$ \\
\hline \multicolumn{8}{|l|}{$\begin{array}{l}\text { Glucose disposal and } \\
\text { insulin secretion }\end{array}$} \\
\hline Fasting plasma glucose & $x$ & $x$ & $x$ & $x$ & $x$ & $x$ & $x$ \\
\hline Fasting insulin and & $x$ & $x$ & $x$ & $x$ & $x$ & $x$ & $x$ \\
\hline \multicolumn{8}{|l|}{ C-peptide } \\
\hline $\mathrm{HbA1c}$ & $x$ & $x$ & & & & & $x$ \\
\hline 3-h OGTT & $x$ & & $x$ & $x$ & $x$ & $x$ & $x$ \\
\hline \multicolumn{8}{|l|}{ Special } \\
\hline Total cholesterol & $x$ & $x$ & & & $x$ & $x$ & $x$ \\
\hline HDL-cholesterol & $x$ & $x$ & & & $x$ & $x$ & $x$ \\
\hline Triglycerides & $x$ & $x$ & & & $x$ & $x$ & $x$ \\
\hline Safety & & $x$ & $x$ & & & & \\
\hline Haematology profile & $x$ & $x$ & $x$ & & $x$ & $x$ & $x$ \\
\hline Chemistry panel & $x$ & $x$ & $x$ & $x$ & $x$ & $x$ & $x$ \\
\hline Serum pregnancy test* & & $x$ & & & & & \\
\hline Total blood samples (ml) & 80 & 80 & 80 & 80 & 800 & 800 & 80 \\
\hline
\end{tabular}

9 and 12) variables collected at baseline will be recorded again, and an OGTT will be performed. AE will be recorded at each follow-up visit.

\section{Study conclusion and further follow-up period}

At the conclusion of the study, a conclusion form will be filled in. The patients will then be tracked up to 5 years after the surgical treatment, assessing lipid and carbohydrate metabolism where possible.

\section{STUDY POPULATION}

\section{Sample size}

The sample size depends on the magnitude of the difference in reactive hypoglycaemia incidence as derived from previous studies. ${ }^{21} 22$ The study is designed to detect a more conservative absolute difference in the occurrence of hypoglycaemia of $50 \%$, expecting an incidence of $70 \%$ in the RYGB group versus an incidence of $20 \%$ in the SG group. A more conservative approach has been chosen, given the limited information available and the inhomogeneous diagnostic criteria used in the different studies. A sample size of 19 patients per group would be required for this clinical trial to detect a difference between treatments at a significance level of 0.05 (twotailed) with a power of 0.90 . Considering an attrition rate of approximately $25 \%$ over the course of the study, a total of 50 subjects ( 25 for each group) will be enrolled.

\section{Study population}

Patients will be recruited from the Obesity Outpatient Clinics and Day Hospital, Catholic University School of Medicine, Rome, Italy. The study will be subject to revision and approval by the Ethical Committee (EC) of the above Institution, in accordance with the guidelines of the National Health Ministry and the Helsinki Declaration, as revised in 2000. All participants will provide written informed consent to participate in the study. Additional written informed consent will be obtained prior to the surgical procedure.

\section{Inclusion criteria}

Patients will be eligible if aged between 25 and 65 years included, if they have a BMI between 35 to greater than $40 \mathrm{~kg} / \mathrm{m}^{2}$ (in the presence of complications such as sleep apnoea, severe coxarthritis or gonarthritis, severe hypertension) and if they are able to understand and comply with the study process.

\section{Exclusion criteria}

- History of type 1 diabetes or secondary diabetes

- Previous bariatric surgery

- History of medical problems such as mental impairment

- Cancer

- Major cardiovascular disease: severe heart valve disease; heart failure; myocardial infarction; endocarditis; atrial fibrillation or flutter; third-degree block

- Major gastrointestinal disease: liver cirrhosis; inflammatory bowel disease; severe hiatal hernia

- Major respiratory disease: pulmonary embolism; pulmonary fibrosis; severe chronic respiratory failure

- Hormonal disorders: pheochromocytoma; Addison's disease; Cushing; Conn's syndrome; Graves's disease; pituitary adenoma with mass effect symptoms or secreting pituitary adenoma

- Infections: HIV; tuberculosis; acute infections 
- History of drug addiction and/or alcohol abuse

- Internal malignancy

- Pregnancy

- Impaired glucose tolerance or type 2 diabetes mellitus

- Suspected or confirmed poor compliance

- Lack of informed consent

- Participants will be excluded if they did not attend at least two initial information visits

\section{TREATMENT}

\section{Surgical program}

Within 1 month of randomisation the patients will undergo RYGB or SG.

\section{Roux-en-Y gastric bypass}

This laparoscopic operation includes the division of the stomach in two parts. A proximal, smaller pouch $(20-25 \mathrm{ml})$, is connected to the rest of the gastrointestinal tract through a gastro-jejunal anastomosis, whereas the distal gastric pouch is left behind, but excluded, from the transit of food.

An entero-entero anastomosis, with a Roux-en-Y type of reconstruction, allows the bile and pancreatic juices to mix with the nutrients at about $100-150 \mathrm{~cm}$ from the gastro-jejunal connection.

\section{Sleeve gastrectomy}

Laparoscopic SG involves a longitudinal resection of the stomach on the greater curvature from the antrum starting opposite of the nerve of Latarjet up to the angle of His. The final gastric volume is about $100 \mathrm{ml}$.

\section{Randomisation process}

Automatic dynamic allocation of the treatment to the patients will be performed via a web-based software. The randomisation procedure via the internet is an automatic and flexible mechanism that on one hand guarantees random allocation and on the other reduces imbalance with respect to the two treatment groups over the two considered stratification factors: gender and age (18-40 and 40-60 years). The procedure may be accessed from any web-connected computer; it clears the user for data access (user id/password); it requires the user to specify the screening number of the subject about to be randomised; it verifies inclusion/exclusion criteria and acquires stratification information; it finally assigns the subject to a treatment, and delivers the corresponding unique randomisation number. The procedure optimises the overall balance of treatments among each stratification cell. The allocation algorithm will be verified by the statistician responsible for the study, who will have continuing access to the randomisation statistics throughout the duration of the study.

\section{Criteria for discontinuation and/or withdrawal \\ - Withdrawal of informed consent \\ - Subject uncooperative}

- Safety reasons as judged by principal investigator

- Non-compliance to the protocol

- Incorrect enrolment

- Subjects who could not be operated laparoscopically

- Conditions requiring medication that could interfere with the outcome of the study, except for those for intercurrent illnesses or adjustment of antihypertensive therapy, as judged by the principal investigator

- Subjects with severe complications due to surgery, as judged by the principal investigator

- Bleeding related to surgery that makes it impossible to draw blood samples

Subjects may be discontinued from the study at any time, at the discretion of the investigator. Subjects are free to discontinue their participation in the study at any time. Subjects who discontinue from the study should always be asked about the reason(s) for the discontinuation. If possible, they should always be seen and assessed by an investigator. AE will be followed up according to Italian Health Ministry guidelines.

If a subject discontinues participation in the study, then her/his enrolment number cannot be issued to another subject.

\section{EFFICACY EVALUATION}

To evaluate the effect of the SG versus the RYGB on glycaemic control the following parameters will be considered.

\section{Primary endpoint}

Primary endpoint: incidence of reactive hypoglycaemia at 1 year after bariatric surgery. According to the American Diabetes Association, a 'true' hypoglycaemic episode is defined as a plasma glucose value $<3.9 \mathrm{mmol} /$ 1 with or without accompanying symptoms. This cut-off will be used after either an OGTT or a glucose profile.

\section{Secondary endpoints}

- Changes at 1 year of insulin sensitivity and insulin secretion during an OGTT

- Changes at 1 year of body weight, BMI, abdominal circumference, body composition and lipid profile

- Incidence of severe hypoglycaemia or related symptoms (shakiness, sweating, dizziness or light-headedness, confusion, difficulty in speaking, weakness, syncope, epilepsy and seizures) assessed by questionnaire and blood glucose measurement (glucometer) or medical report within 5 years after surgery

\section{Evaluation methods and timing}

During the experimental study the following clinical evaluations will be performed:

A. Demographic data (month 3)

- Date of birth

- Gender

- Ethnicity 
B. Medical and surgical history (month 3, month 1)

C. Anthropometric measurements (month 3, month 1, months 1, 3, 6, 9 and 12)

- Body weight (will be measured to the nearest $0.1 \mathrm{~kg}$ on a balanced beam scale in the morning before breakfast after a visit to the lavatory, with the subjects in their underwear).

- Height (will be measured to the nearest $0.5 \mathrm{~cm}$ using a stadiometer)

- Waist circumference (will be measured at the part of the trunk midway between the most caudal part of the lateral costal arch and the iliac crest in the morning before breakfast, after lavatory, visit with the person standing with feet about $25-30 \mathrm{~cm}$ apart. The measurer will stand beside the individual and fit the tape snugly, without compressing any underlying soft tissues. The circumference will be measured to the nearest $0.5 \mathrm{~cm}$, at the end of a normal expiration.

- Hip circumference (will be measured as the maximal circumference over the buttocks)

- BMI (will be computed as weight $(\mathrm{kg}) /$ height $\left(\mathrm{m}^{2}\right)$ )

D. Physical examination (month 3 , month 1 , months 1 , $3,6,9$ and 12)

- General appearance

- Skin

- Head and neck

- Lymph nodes

- Thyroid

- Cardiovascular system

- Respiratory system

- Abdomen

- Other

E. Cardiovascular parameters (month 3, month 1, months 1, 3, 6, 9 and 12)

- Blood pressure

- Heart rate

- (blood pressure and heart rate will be measured in sitting position in duplicate after 15 min rest)

- ECG (recorded under resting conditions. It will be performed according to standard routine at bariatric surgery)

F. Biochemical analysis (month 3, month 1 , months 1 , $3,6,9$ and 12)

- Fasting plasma glucose

- Fasting plasma insulin

- Fasting plasma C-peptide

- HbAlc

- Total cholesterol

- High-density lipoprotein cholesterol

- Triglycerides

- Haematology profile

- Chemistry panel

G. OGTT (month 3, month 1, months 1, 3, 6, 9 and 12) At 8:00-9:00 h, after a $12 \mathrm{~h}$ overnight fast, an intravenous catheter is placed in one antecubital vein to draw blood samples. A $75 \mathrm{~g}$ of oral glucose bolus is administered in $10 \mathrm{~min}$ maximum and blood samples are obtained at $-20,-5,5,10,20,30,40,60$, $80,100,120,140,160$ and $180 \mathrm{~min}$ relative to the administration of the bolus. Samples are placed in heparinised tubes, and plasma is separated within $20 \mathrm{~min}$ and stored at $-70^{\circ} \mathrm{C}$.

$\mathrm{H}$. The patients will be asked to monitor their blood glucose by a seven-point glucose profile (ie, before and $2 \mathrm{~h}$ after breakfast, lunch and dinner plus one control before bedding) randomly performed once a week.

\section{Analytical methods}

Plasma glucose will be measured by the glucose-oxidase method (Beckman, Fullerton, California, USA). Plasma insulin will be assayed by microparticle-enzyme immunoassay (Abbott, Pasadena, California, USA) with a sensitivity of $1 \mu \mathrm{U} / \mathrm{ml}$ and an intra-assay $\mathrm{CV}$ of $6.6 \%$.

C-peptide will be assayed by radioimmunoassay (MYRIA; Technogenetics, Milan, Italy): minimal detectable concentration $=17 \mathrm{pmol} / 1$ and interassay and intra-assay CVs of 3.3-5.7\% and 4.6-5.3\%, respectively.

\section{Mathematical modelling}

Empirical indices of insulin sensitivity ${ }^{24}$ and secretion $\left(\mathrm{AUC}_{\mathrm{I}} / \mathrm{AUC}_{\mathrm{G}}\right.$ (area under the curve) ratio) will be computed. Glucose and insulin concentrations will be fitted by the oral glucose minimal model after the OGTT. Insulin secretion will also be computed via a deconvolution approach on C-peptide measurements.

A. DEXA variables (month 1 , months 6,9 and 12)

- Body composition will be measured by DEXA (Lunar Prodigy), which provides results on total and regional (trunk, arms, legs, pelvis) fat mass, fat-free mass and bone mass. The following will be recorded:

- Total body bone mineral density

- Lean body mass

- Fat mass

- Percent weight body fat

B. Concomitant medications (month 3, month 1, months 1, 3, 6, 9 and 12)

\section{SAFETY AND TOLERANCE EVALUATION AND ASSESSIMENT}

The safety of the surgical procedures will be evaluated at the end of the study, analysing all the information recorded in the Case Report Form (CRF) designed for the study, according to the timing and modalities described in other sections of the present protocol.

Information to assess safety will include: objective exam, patient's symptoms related to the operation, occurrence of AE.

\section{Safety parameters}

\section{Adverse events}

The clinical tolerability will be evaluated by recording the occurrence of $\mathrm{AE}$ as reported by the patient or observed by the medical investigator. Any AE occurring after surgery will have to be recorded in the CRF. 


\section{Definitions}

An AE) is any untoward medical occurrence in a patient or clinical investigation subject undergoing a treatment and which does not necessarily have a causal relationship with this treatment. An AE can therefore be any unfavourable and unintended sign, symptom or disease temporally associated with the use of a medicinal product, or related to the surgical procedure. Preexisting events, which increase in frequency or severity, or change in nature during or as a consequence of use of a drug in human clinical trials, will also be considered AEs.

A serious adverse event (SAE) is defined as any $\mathrm{AE}$ regardless of causality that

- Leads to death.

- Leads to a serious deterioration in the health of the subject that

- results in life-threatening illness or injury,

- results in permanent impairment of a body structure or a body function,

- requires inpatient hospitalisation or prolongation of existing hospitalisation.

- Results in medical or surgical intervention to prevent permanent impairment to a body structure or a body function.

- Leads to fatal distress or death.

\section{Adverse event reporting procedures}

If the investigator identifies the occurrence of an $\mathrm{AE}$ or SAE, the corresponding report form must be completed and transmitted to the coordinating investigator within $24 \mathrm{~h}$. The form will be part of the study documentation. Any fatal or life-threatening event should be reported immediately to the coordinating investigator: this preliminary report will be followed within $24 \mathrm{~h}$ by detailed documentation including the completed SAE form, copies of hospital case reports, autopsy reports and other documents, when requested and applicable.

Minimal information contained in the AE/SAE report form should include:

- Identification of the subject or patient

- Type of surgical treatment

- An identifiable reporting source

- All related AE

- All medications used

Follow-up of SAEs that occur during the study will continue until satisfactory resolution or stabilisation, as judged by the investigator. The coordinating investigator may request that certain AE be followed until resolution.

If and when supplementary information is available, a follow-up SAE Report Form must be completed by the investigator and delivered within $24 \mathrm{~h}$ to the coordinating investigator.

The coordinating investigator must inform the Ethics Committee if the serious AE is likely to affect the safety of the subjects or the conduct of the study.

Moreover, it will be the responsibility of the coordinating investigator to inform in writing all clinical investigators about all serious AE occurring during the study. This information shall be sent to the clinical investigators based on perceived risk.

\section{DIRECT ACCESS TO ORIGINAL DOCUMENTATION}

The investigator will allow the national Regulatory Authority, and the staff possibly indicated by the EC or by coordinating investigator, direct access to the complete original documentation, including informed consent and the clinical records or outpatient registers. People who have direct access to this documentation will have to take all reasonable precautions to avoid disclosure of the patient identity and of all information which is the property of the coordinating investigator, in compliance with applicable laws.

\section{QUALITY ASSURANCE PROCEDURES}

The organisation, monitoring and quality assurance of the present study will be under the responsibility of the coordinating investigator.

\section{Clinical monitoring}

The clinical monitoring will be carried out by qualified persons assigned by the coordinating investigator and will be conducted according to the guidelines of the ICH GCP. In addition, the monitoring activities will include the verification of the correct completion of the CRFs and, when applicable, the consistency between source documents and stored electronic data are used for the randomisation procedures. The coordinating investigator will ensure the practical training for the personnel involved in the study.

\section{Data review and audits}

Data review and audits will be carried out by qualified persons assigned by the coordinating investigator.

The CRFs will be tracked and periodically updated.

Moreover, audit visits to Hospital wards may be planned in order to verify that:

- Compliance with the protocol is maintained

- Any deviation from the protocol are discussed with the coordinating investigators and documented

- Study staff and facilities are qualified to conduct the study safely and effectively

- Informed consent forms have been obtained

- The procedures for recording and reporting $\mathrm{AE}$ are followed

- EC approvals have been obtained and protocol amendment notifications have been carried out

- Randomisation procedures have been followed

- Subject withdrawals have been documented and discussed with the coordinating investigator

The audit is a check of the study activities and documentation aimed at verifying if the study activities have been conducted and the data have been recorded and transmitted in accordance with the protocol, with the GCPs, with institutional SOPs and with applicable laws. 
Audit visit reports will be produced and communicated to the study investigators and to thec.

\section{CRF structure}

For the investigator three main phases will be foreseen: enrolment of a new patient (at the screening visit), randomisation procedure (at the basal visit), further data insertion of a randomised patient's data (at the follow-up visits). For the first and third phase a standard paper CRF will be completed. The randomisation procedure will be carried out by means of an electronic procedure.

- Enrolment of a new patient. In this phase preliminary information will have to be recorded (such as patient screening number, gender, age, date of admission, concomitant medications and the presence of risk factors) in order to assess the eligibility of the patient and to include her/him in the study protocol. Each time an investigator will admit a new patient a consecutive screening number will be assigned.

- Randomisation procedure. After the patient's demographic and basal characteristics have been collected, a randomisation procedure will be performed by an electronic system according to the procedure described above. After entering the patient screening number and the information needed for stratification, the treatment will be randomly assigned by the system.

- Data recording. The investigators will record all subjects' study data in a paper CRF identified with that patient's screening number.

All necessary documentation containing the instructions for the web access and for the electronic randomisation procedure will be provided to the investigators prior to the beginning of the study.

\section{Data management}

The verification of the experimental data will be made according to the following procedure:

- The monitor will verify the correct and complete transcription of the experimental data in the CRF and will verify the correspondence with the source documents in the Experimental Centre;

- If necessary, queries will be generated, and changes and/or comments will be required of the investigators.

\section{STATISTICAL ANALYSIS}

\section{Study populations}

There are two analysis populations, which will be used in evaluating treatment differences: intent-to-treat (ITT) (which coincides with the safety-analysable population) and the efficacy-evaluable (completer-complier or per-protocol, EE).

The ITT population consists of all randomised patients.
The EE population consists of all patients who completed the study, that is, all patients where the primary endpoint was measured.

\section{Efficacy analysis}

\section{Primary efficacy analysis}

The primary analysis will test if the SG surgical treatment determines a lower incidence of reactive hypoglycaemia than the RYGB surgical treatment. The analysis will be performed by means of Fisher's exact test. The type I error will be set at 0.05 (two-tailed).

\section{Secondary efficacy analyses}

The statistical analyses on the secondary endpoints will have an explorative character, so, for each analysis, the type I error will be set to the nominal level of 0.05 .

All the categorical variables (binary or with more than two modalities) will be analysed by means of a $\chi^{2}$ test or a Fisher exact test when appropriate. For continuous variables relative $\delta$ s will be computed (as difference between values at 1 year and values at baseline, over basal values) and will be analysed by means of a test for independent samples or an equivalent nonparametric test as appropriate. Differences between groups and trends over time will be analysed by means of an analysis of variance for repeated measurements.

\section{Safety and tolerability analysis}

All patients who undergo the surgical treatment will be included in the safety analysis. Safety analyses will include tabulation of type and frequency of any recorded AE. Between-group differences in the proportion of patients reporting an $\mathrm{AE}$ will be analysed using Fisher's exact test whereas all continuous safety laboratory evaluations will be summarised using descriptive statistics, and relative $\delta$ s will be computed as above. Relative $\delta$ s will be analysed by means of the Mann-Whitney U test or the $t$ test as appropriate.

\section{ETHICAL ASPECTS}

All the involved parts in the study agree and will verify that the experimental study is conducted in compliance with ethical principles originating from the Helsinky Declaration, with the guidelines of Good Clinical Practice (GCP) and with applicable laws.

\section{Ethical authorisations}

The coordinating investigator has the responsibility of submitting the present clinical protocol to the local EC for approval, before enrolling patients. The coordinating investigator will have to provide the EC with all necessary documents for obtaining approval.

\section{Informed consent}

Before the start of the study, the Informed Consent forms to be provided to the patients will be submitted to the EC for examination and approval, along with the 
protocol. The signed Informed Consent will be obtained after having informed the patient of all aspects of the trial as reported in the guidelines of the GCP.

\section{ADMINISTRATIVE PROCEDURES \\ Changes in the study conduction or in the planned analyses}

Each change in the conduction of the study is defined 'Amendment to the Clinical Protocol'. This term is referred to any change brought to the experimental protocol after the EC has given its final approval.

Amendments to the protocol are changes of a legal document which has a binding value and therefore must be approved in writing and signed in two copies by the same signatories of the protocol.

All amendments to the protocol must be submitted to the assessment of the EC who approved the study protocol, before being applied.

The coordinating investigator has the responsibility of submitting the amendment to the EC in order to obtain an approval in writing. The approval will be delivered and kept with the same modalities as the study protocol.

When changes are referred only to administrative or logistic aspects of the study, the related amendment must simply be notified to the EC.

It must be considered that whenever the amendment significantly alters the study design or the potential risks to which patients are exposed, each patient will have to be informed and will have to put down in writing her/his intention to continue the study. An appropriate form will be prepared by the coordinating investigator and will be approved by the EC.

\section{Study suspension/interruption}

Complete suspension of the study

The coordinating investigator will have the power of suspending/interrupting the study whenever:

The rate of patient enrolment will be shown to be ineffective:

- The clinical investigators do not respect the requirements of the study, particularly where the evaluation of the patient inclusion/exclusion criteria is concerned.

- The Experimental Centre is not capable of meeting the requirements specified in the guidelines of GCP (GCP-ICH DM n. 162 del 15/07/97):

The coordinating investigator will inform the EC of the early interruption of the study, justifying the decision made.

\section{Suspension of the study by the EC}

The study can also be suspended by the EC. A detailed explanation in writing of the conclusion or suspension of the study will be provided.

\section{Archiving}

The coordinating investigator is responsible for the conservation of the study documents in compliance with applicable laws. The coordinating investigator will take all measures necessary to avoid accidental or early document destruction.

\section{Confidentiality}

Individual subject medical information, obtained as a result of this study, is considered confidential and disclosure to third parties is prohibited. Such medical information may be given to the subject's physician or to other appropriate medical staff responsible for the subject's well-being.

\section{Financing of the study}

No financial aspects must be taken into account because no funding is foreseen for the present study.

\section{RESPONSIBILITY OF THE INVESTIGATOR}

The investigators are responsible towards the coordinating investigator for all actions carried out by their staff involved in the conduction of the study. Except when specifically required, the term 'investigator' used in this protocol and in the CRF is referred to the investigator or to a qualified person designed by him/her to carry out all the activities related to the clinical trial and to sign in his/her stead the study documents. The investigator must be responsible for the safety and well-being of the human subjects involved in the clinical investigation and must conduct the study in compliance with the study protocol, and with the Helsinky Declaration (1964) and subsequent revisions.

\section{FINAL REPORT OF THE STUDY}

Within 3 months from the end of the study, a Study Final Clinical Report, including the clinical comments based on the results of the statistical analysis, will be drafted by the coordinating investigator and/or by a delegated person.

Contributors GM, SP, AG, RB and MR drafted the article. CPL, CC and CG revised the article critically for important intellectual content. All the authors approved the final version to be published.

Funding This research received no specific grant from any funding agency in the public, commercial or not-for-profit sectors.

Competing interests None.

Ethics approval Catholic University Ethical Committee.

Provenance and peer review Not commissioned; externally peer reviewed.

\section{REFERENCES}

1. Flegal KM, Carroll MD, Kit BK, et al. Prevalence of obesity and trends in the distribution of body mass index among US adults, 1999-2010. JAMA 2012;307:491-7.

2. Sacks FM, Bray GA, Carey VJ, et al. Comparison of weight-loss diets with different compositions of fat, protein, and carbohydrates. N Engl J Med 2009;360:859-73. 
3. Gastrointestinal Surgery for Severe Obesity. NIH Consens Statement 1991;9:1-20.

4. Steinbrook R. Surgery for severe obesity. N Engl J Med 2004;350:1075-9.

5. Mingrone G, Panunzi S, De Gaetano A, et al. Bariatric surgery versus conventional medical therapy for type 2 diabetes. $N$ Engl $J$ Med 2012;366:1577-85.

6. Schauer PR, Kashyap SR, Wolski K, et al. Bariatric surgery versus intensive medical therapy in obese patients with diabetes. $N$ Engl $J$ Med 2012;366:1567-76.

7. Sjöström L, Lindroos AK, Peltonen $M$, et al. Lifestyle, diabetes, and cardiovascular risk factors 10 years after bariatric surgery. $N$ Engl $J$ Med 2004;351:2683-93.

8. Livingston $\mathrm{EH}$. The incidence of bariatric surgery has plateaued in the U.S. Am J Surg 2010;200:378-85.

9. Regan JP, INabnet WB, Gagner M, et al. Early experience with two-stage laparoscopic Roux-en-Y gastric bypass as an alternative in the super-super obese patient. Obes Surg 2003;13:861-4.

10. Deitel M, Crosby RD, Gagner M. The first international consensus summit for sleeve gastrectomy (SG), New York City, 25-27 October 2007. Obes Surg 2008;18:487-96.

11. Lee CM, Cirangle PT, Jossart GH. Vertical gastrectomy for morbid obesity in 216 patients: report of two-year results. Surg Endosc 2007;21:1810-16.

12. Himpens J, Dapri G, Cadiere GB. A prospective randomized study between laparoscopic gastric banding and laparoscopic isolated sleeve gastrectomy: results after 1 and 3 years. Obes Surg 2006;16:1450-6.

13. Langer FB, Hoda Reza MA, Bohdjalian A, et al. Sleeve gastrectomy and gastric banding: effects on plasma ghrelin levels. Obes Surg 2005:15:1024-9.

14. Salinari S, Bertuzzi A, Guidone $C$, et al. Insulin sensitivity and secretion changes after gastric bypass in normotolerant and diabetic obese subjects. Ann Surg (in press)
15. Laferrère $\mathrm{B}$, Heshka $\mathrm{S}$, Wang $\mathrm{K}$, et al. Incretin levels and effect are markedly enhanced 1 month after Roux-en- $Y$ gastric bypass surgeryin obese patients with type 2 diabetes. Diabetes Care 2007:30:1709-16.

16. Laferrère B, Teixeira J, McGinty $\mathrm{J}$, et al. Effect of weight loss by gastric bypass surgery versus hypocaloric diet on glucose and incretin levels in patients with type 2 diabetes. J Clin Endocrinol Metab 2008;93:2479-85.

17. Service GJ, Thompson GB, Service FJ, et al. Hyperinsulinemic hypoglycemia with nesidioblastosis after gastric-bypass surgery. N Engl J Med 2005;353:249-54

18. Patti ME, McMahon G, Mun EC, et al. Severe hypoglycaemia post-gastric bypass requiring partial pancreatectomy: evidence for inappropriate insulin secretion and pancreatic islet hyperplasia (see comment). Diabetologia 2005;48:2236-40.

19. Engstrom Eden B, Burman P, Holdstock $C$, et al. Effects of gastric bypass on the GH/IGF-I axis in severe obesity —and a comparison with GH deficiency. Eur J Endocrinol 2006;154:53-9.

20. Carpenter T, Trautmann ME, Baron AD. Hyperinsulinemic hypoglycemia with nesidioblastosis after gastric-bypass surgery. N Engl J Med 2005;353:2192-4.

21. Roslin M, Damani T, Oren J, et al. Abnormal glucose tolerance testing following gastric bypass demonstrates reactive hypoglycemia. Surg Endosc 2011;25:1926-32.

22. Tzovaras G, Papamargaritis D, Sioka E, et al. Symptoms suggestive of dumping syndrome after provocation in patients after laparoscopic sleeve gastrectomy. Obes Surg 2012;22: 23-8.

23. Marsk R, Jonas E, Rasmussen F, et al. Nationwide cohort study of post-gastric bypass hypoglycaemia including 5040 patients undergoing surgery for obesity in 1986-2006 in Sweden. Diabetologia 2010;53:2307-11

24. Matsuda M, DeFronzo RA. Insulin sensitivity indices obtained from oral glucose tolerance testing. Diabetes Care 1999;22:1462-70. 